\title{
Effect of Concurrent Strength and Endurance Training Sequence on Muscular and Aerobic Fitness for Volleyball Players
}

\author{
Amr Hassan Tammam ${ }^{1}$ \\ Enas Mohamed Hashem ${ }^{2}$
}

\author{
${ }^{1}$ Professor of Sports Training at the Department of Training and Sports Movement Sciences, Faculty of Physical Education, \\ Tanta University, Egypt \\ ${ }^{2}$ Lecturer of Volleyball at the Department of Games and Racquet Sports, Faculty of Physical Education, Tanta University, \\ Egypt
}

\begin{abstract}
The aim of current study was to examine the effect of 8-week concurrent strength and endurance training with different intra-session sequence; strength-endurance sequence (SES), endurance-strength sequence (ESS), and alternation sequence between sessions (ASBS) on muscular and aerobic fitness for volleyball players. Twenty volleyball players from Tanta club volunteered to participate in current study. They were distributed into three different concurrent training (CT) intra-session sequence groups; SES $(n=7), \operatorname{ESS}(n=7)$, and ASBS $(n=6)$, they were tested before training program (PRE) and after 8-week CT (POST) for one repetition maximum-1RM (i.e. squat and bench press), local muscular endurance (LME) (i.e. squat and bench press), and $4 \mathrm{~km}$ running time trail. There were statistically significant effects of the time (i.e. pre- to post-training) for all measured variables $(p \leq 0.05)$ of all three groups SES, ESS, and ASBS. Results showed that there was statistically significant differences favor SES group $(p \leq 0.05)$ in $1 R M$ of squat and bench press in comparison with the ESS group. There were statistically significant differences $(p \leq 0.05)$ favor SES group in LME of Squat comparison with the ESS group, no statistically significant differences $(p>0.05)$ were found between SES, ESS, and ASBS in LME of bench press. There was significant differences favor ESS group $(p \leq 0.05)$ in $4 \mathrm{~km}$ running time trail of in comparison with the SES group. In Conclusion, The concurrent strength and endurance training with different intersession sequence improved muscular and aerobic fitness.
\end{abstract}

Key words: Simultaneous performance, interference phenomenon, sequence

\section{Introduction}

$\mathrm{V}$ olleyball is a multifaceted team-sport that demands a range of physical qualities, including endurance, strength, power, etc. Developing of these qualities simultaneously is a challenge for coaches and practitioners, especially in the pre-season period when players typically return to training after a period of rest in a de-conditioned state (Marques, 2009). The combine strength and endurance training within the same training cycle has been defined as CT (Fyfe, Bishop, \& Stepto, 2014). Concurrent strength and endurance training have the potential to bring about gains in muscular fitness and cardiorespiratory simultaneously.(Kang \& Ratamess, 2014).

Previously, it has been documented that CT can result in compromised muscle strength and (or) power capacity (Wilson et al., 2012). This blunted response in strengthrelated adaptation has been referred to as the 'interference phenomenon' (Hickson, 1980). The compatibility of strength and endurance training has been investigated over the last 3 decades; authors have proposed a variety of hypothesis to explain this phenomenon, these include; an inability to adapt as a consequence of contrasting training intensities (Docherty \& Sporer, 2000), an incompatible hormonal (Bell, 2000) and (or) molecular signaling environment (Atherton et al., 2005) the 'sequence' or 'order' of training (Moktar Chtara et al., 2008; Enright, 2015), the recovery time between training bouts (Alves, 2015; Quebedeaux, 2007) and the frequency of aerobic training (Wilson et al., 2012). Studies have reported that CT could hinder aerobic adaptations and attenuation of strength development because of an inhibitory effect on muscle (García-Pallarés \& Izquierdo, 2011). Afterwards, it was reported that $\mathrm{CT}$ impairs the development of strength and muscular power but did not affect the development of aerobic capacity compared to both forms of stand-alone training (Izquierdo \& Exposito, 2010; Santos \& Izquierdo, 2012). Nevertheless, some studies have shown no antagonistic effects on strength (McCarthy, 2002) or aerobic performance (Mikkola, 2007) following CT. It seemed that the physiological adaptations that followed CT are dependent on the type and degree of the stimulus applied during the training session (Baar, 2006; Silva et al., 2012), the incorporation of recovery post training (Alves, 2015; Quebedeaux, 2007), and order of stimulus applied during training session (Moktar Chtara et al., 
2008; Enright, 2015). Understanding the physiological responses to the CT practices may be important, this may lead to a better understanding of how to minimize the interference phenomenon and make the training stimuli more effective.

A unique perspective of CT is the sequence or order in which endurance and strength exercise are presented when performed simultaneously within the same session. Most studies that have compared the efficacy of two exercise orders generally suggest that the intra-session sequence of training produces no significant differences in traininginduced adaptations between groups because both combinations allow similar improvements in cardiorespiratory and muscular (Moktar Chtara et al., 2008; Enright, 2015; Gravelle \& Blessing, 2000; McGawley \& Andersson, 2013). However, some of these studies also have indicated that each training sequence could have its own unique advantages and drawbacks that one should consider to pursue CT more effectively (Kang \& Ratamess, 2014; McCarthy, 2002; Mikkola, 2007). At present, there is no consensus as to which exercise sequence is more effective, but sequencing recommendations are dependent on the program priorities.

To date there does not seem to be any study have investigated the effect of CT sequence in Egyptian volleyball players, moreover, it is the first study tried to investigate the effect of alternation sequence between workout/ sessions on muscular and aerobic fitness. Knowledge gained through this study could help to make CT workouts more effective without having to isolate strength and endurance training on separate days. Thus, volleyball players could improve their workout without adding time or energy.

So the aim of current study was to examine the effect of 8week concurrent strength and endurance training with different intra-session sequence; strength-endurance sequence (SES), endurance-strength sequence (ESS), and alternation sequence between sessions (ASBS) on muscular and aerobic fitness for volleyball players. Authors hypothesized that (1) muscular and aerobic fitness would increase in all training groups that performed CT, (2) the SES group would have more gains in muscular fitness than the other two groups, (3) the ESS group would have more gains in aerobic fitness than the other two groups.

\section{Material and Methods}

\section{Subjects:}

Twenty volleyball players from Tanta club volunteered to participate in current study. They were distributed in a balanced manner according to their Pre values of study variables into three different $\mathrm{CT}$ intra-session sequence groups; strength and endurance sequence (SES) $(n=7)$, endurance and strength sequence $(\mathrm{ESS})(\mathrm{n}=7)$, and alternation the two sequence between sessions (ASBS) ( $n$ $=6$ ). The inclusion criteria for the participation were no use of any ergogenic supplements, not participate in any regular resistance or endurance training during the study, and If participants missed more than 1 training session, they were removed from the study. The three training groups showed no significant differences for pretraining characteristics $(\mathrm{p}>0.05)$ present in (Table 1$)$.

\section{Experimental Design:}

Timing of the study started on June, 2015 (off-season period), subjects were tested before training program (Pre) and after 8-week CT (Post) for lower and upper-body one repetition maximum-1RM (i.e. squat and bench press), local muscular endurance (LME) (i.e. squat and bench press), and $4 \mathrm{~km}$ running time trail (Figure 1).

Table 1

Subjects' characteristics*

\begin{tabular}{|c|c|c|c|c|c|c|c|c|}
\hline \multirow{2}{*}{ Variables } & \multicolumn{2}{|c|}{ SES (n = $)$} & \multicolumn{2}{c|}{ ESS (n =7) } & \multicolumn{2}{c|}{ ASBS (n=6) } \\
\cline { 2 - 8 } & Mean & \pm SD & & Mean & \pm SD & & Mean & \pm SD \\
\hline Age (y) & 23.29 & 2.50 & & 24.57 & 4.20 & & 25 & 1.05 \\
Weight (kg) & 81.57 & 4.24 & & 82.43 & 4.43 & 82.17 & 3.31 \\
Height (m) & 1.85 & 0.07 & & 1.86 & 0.06 & 1.88 & 0.08 \\
Body mass index (kg/m2) & 23.74 & 1.02 & & 23.82 & 1.45 & 23.36 & 1.67 \\
\hline
\end{tabular}

* $\mathrm{SES}=$ strength and endurance sequence; ESS= endurance and strength sequence; ASBS alternation sequence between sessions. 
Figure 1

Experimental design

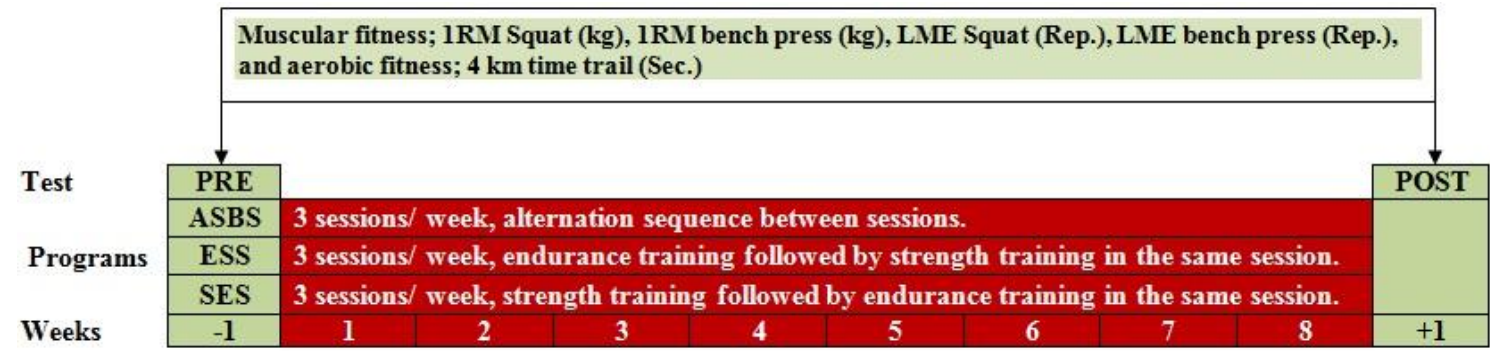

One week before the start of experiment, all subjects report to the Tanta club gym 4 times in non-consecutive days. On the 1st visit; all subjects were provided with an information sheet setting out details of the experiment, and written informed consent were completed and signed. Body weight and height were recorded using a balance weighing scales and portable stadiometer respectively, then they lied down on backs for 15 min to measure the heart rate at rest (HRrest) by Polar ${ }^{\circledR}$ heart rate monitors (Polar, M400, France). After warm up, subjects completed a resistance training session with no or little resistance, this session familiarized the subjects with the equipments and proper exercise techniques. Two days after the 1st visit of familiarization session, all subjects performed $1 \mathrm{RM}$ test and randomly performed the other 2 tests (i.e. LME or aerobic test) in 2 non-consecutive days. All testing sessions for 3 groups used the same test order, equipment, warm-up, and time of the tests (between 5:00 and 8:00 PM). The post assessment was performed for each group after 48hrs from last session in the training program. All testing and training sessions were supervised and conducted by the same authors, in addition to 2 certified fitness trainers.

\section{Muscular Fitness measurements:}

Maximal strength test, 1RM was used as a measure of maximal strength, all subjects performed one trail of 1RM for 2 exercises previously mentioned, in addition to the strength program exercise that included (leg curl, standing calf raise, lat pull-down, military press, and arm curl) to determine the beginning loads $(\mathrm{kg})$ for subjects' resistance training programs. Before the test the subjects performed a warm-up which included 5 min of light treadmill running and 5 min stretching exercises. Thereafter, the subjects performed 10 repetitions at a relatively light load that served as a specific warm-up, followed by a gradual increase in load until 1RM was achieved (Baker, Wilson, $\&$ Carlyon, 1994). The rate of the gradual increase in load was dependent on the participant's self-perceived capacity, and it ranged from 1 to $10 \mathrm{~kg}$, with $3-5$ min rest interval between attempts, and the 1RM was achieved within 3-5 attempts. The Authors provided encouragement to all subjects during testing, in an attempt to elicit a maximal effort.

The local muscular endurance (LME) test consisted of the maximal number of repetitions achieved with $70 \%$ of the 1 RM load on the squat and bench press. For this test, the participants performed the same warm-up procedures as in the 1RM test (Silva et al., 2012). The post-training LME measurements were performed at the same relative load of PRE value and compared to the pre-training measurements.

\section{Aerobic fitness test:}

A $4 \mathrm{~km}$ running time trail was used as a measure of aerobic fitness/ performance; this test consisted of covering $4 \mathrm{~km}$ in the minimum time on a $400 \mathrm{~m}$ outdoor athletic track (M Chtara et al., 2005), monitoring by stopwatch Casio® (Casio HS-80TW, Japan). The test was preceded by a standardized 10 minute warm up period. Subjects of each group performed the test together.

\section{Concurrent training programs:}

Subjects of each group took part in a CT program that lasted 8 weeks included strength and endurance exercises with different intra-session sequence. The SES group trained on Saturday, Monday and Wednesday, all sessions started with strength training followed by endurance training, whereas the ESS group trained on Sunday, Tuesday and Thursday, all sessions started with endurance training followed by strength training, while the ASBS group trained on Monday, Wednesday and Friday, with different sequence of concurrent strength and endurance training each session. The rest interval between strength and endurance training or vice versa was 10 min included moving from the gym to the running track or vice versa and performing stretching exercises. 
Table 2

Strength training program for all groups*

\begin{tabular}{|c|c|c|c|c|c|}
\hline Cycles & \multicolumn{2}{|c|}{ Mesocycle 1 } & \multicolumn{3}{|c|}{ Mesocycle 2 } \\
\hline Duration of cycle (weeks) & \multicolumn{2}{|c|}{ Strength endurance } & \multicolumn{3}{c|}{ Maximal strength } \\
Main objectives & $1 \& 2$ & $3 \& 4$ & & $5 \& 6$ & $7 \& 8$ \\
\hline Weeks & $75 \%$ & $80 \%$ & $85 \%$ & $90 \%$ \\
Intensity (\% 1RM) & $12-15$ & $8-10$ & $5-6$ & $3-4$ \\
Repetitions & 3 & 3 & 3 & 2 \\
Number of sets & 3 & 3 & $2: 1: 2$ & $1: 1: 1$ & $1: 1: 1$ \\
Rest intervals (min) & $2: 1: 2$ & & 2 & 2 \\
Repetitions velocity (sec.) & Squat, Bench press, Leg curl, Lat pulldown, Standing calf raise, Military press, Abdominal \\
\hline
\end{tabular}

$* 1 \mathrm{RM}=$ One repetition maximum

Strength training program were designed based on previous studies published in the literature (M Chtara et al., 2005; Glowacki et al., 2004; Kang \& Ratamess, 2014; Silva et al., 2012). It was divided into two mesocycles of four weeks each. The general objective for mesocycles 1 and 2 were development of strength endurance, and maximal strength, respectively. A percentage of each subject's 1RM for each exercise was used to determine the intensity. The intensity and number of repetitions performed for each exercise were progressively changed biweekly. A more detailed description of the progression of the strength-training program is presented in (Table 2)

Endurance training program were designed based on previous studies published in the literature (Moktar Chtara et al., 2008; Glowacki et al., 2004; Janssen, 2001; Kang \& Ratamess, 2014). It was divided into two training zones (i.e. A1 and A2) of four weeks each. A1 and A2 objectives were development of aerobic fitness using continues training method. The running intensity was determined by a percentage of heart rate reserve (HRR) calculated according to Karvonen equation (Karvonen, 1957). Training sessions lasted between 20 and $45 \mathrm{~min}$, and exercise heart rates were continuously monitored using Polar® heart rate monitors. The intensity and/or duration of each session were increased biweekly as training progressed. A more detailed description of the progression of the endurance-training program is presented in (Table 3)

Table 3

Endurance training program for all groups*

\begin{tabular}{|c|c|c|c|c|}
\hline Training Zone & \multicolumn{2}{|c|}{ A1 } & \multicolumn{2}{|c|}{ A2 } \\
\hline $\begin{array}{c}\text { Duration of zone (weeks) } \\
\text { Main objectives } \\
\text { Energy source }\end{array}$ & \multicolumn{2}{|c|}{$\begin{array}{c}4 \\
\text { Aerobic endurance } \\
\text { Fats }\end{array}$} & \multicolumn{2}{|c|}{$\begin{array}{c}4 \\
\text { Aerobic endurance } \\
\text { Glucose } \\
\end{array}$} \\
\hline Weeks & $1 \& 2$ & $3 \& 4$ & $5 \& 6$ & $7 \& 8$ \\
\hline $\begin{array}{c}\text { Intensity (\% HRR) } \\
\text { HR zone (beat) } \\
\text { Duration (min) }\end{array}$ & $\begin{array}{c}70 \% \\
120-130 \\
20-25\end{array}$ & $\begin{array}{c}75 \% \\
130-140 \\
25-30\end{array}$ & $\begin{array}{c}75 \% \\
140-150 \\
35-40\end{array}$ & $\begin{array}{c}80 \% \\
150-160 \\
40-45\end{array}$ \\
\hline Exercises & \multicolumn{4}{|c|}{ Continuous running on $400 \mathrm{~m}$ out-door track } \\
\hline
\end{tabular}

$*$ HRR $=$ Heart rate reserve

\section{Statistical analysis:}

All data are presented as mean and standard deviations $( \pm \mathrm{SD})$. The statistical calculations were performed using STATISTICA software version 10 (StatSoft, Tulsa, OK, USA). All variables presented normal distribution and homoscedasticity. The 1RM, LME and aerobic test were evaluated separately, so one-way analysis of variance (ANOVA) was used to test for significant differences between groups, and when statistical significance $(\mathrm{P} \leq$ 0.05) was found, the Scheffe post hoc test for comparisons was applied to compare the difference between groups in PRE, and POST time points. To test for significant differences between PRE and POST variables for each group, paired sample t-test was used.

\section{Results}

There were no differences between the three groups in the PRE test for all study variables $(p>0.05)$. All subjects completed the 8-week training with attendance rate more than $95 \%$, with only 6 participants absent for 1 training session (SES = 1 player; ESS $=3$ players; ASBS $=2$ players) during the 8 -week training programs. There were statistically significant effects of the time (i.e. pre- to posttraining) for all measured variables $(p \leq 0.05)$ of all three groups (i.e. SES, ESS, and ASBS) (Table 4). 


\section{Muscular fitness}

1RM: The study has shown statistically significant differences favor SES group $(p \leq 0.05)$ at the POST evaluation in 1RM of squat and bench press $(22.47 \%$, and $15.43 \%$; respectively) in comparison with the ESS group (13.01\%, and $7.72 \%$; respectively), whereas there is no statistically significant differences $(p>0.05)$ were found in comparison with the ASBS group (17.01\%, and 9.33\%; respectively). Also results has shown no statistically significant differences $(p>0.05)$ between ASBS and ESE in 1RM of squat and bench press at POST evaluation, however the ASBS group shown a higher percentage increase in maximal strength than ESS group for those exercises (Table 4) (Fig. 2A).

Table 4

Pre- and post-testing data for each variable

\begin{tabular}{|c|c|c|c|c|c|}
\hline \multirow{2}{*}{ Variable } & \multirow{2}{*}{ Group } & \multicolumn{2}{|c|}{ PRE } & \multicolumn{2}{c|}{ POST } \\
\cline { 2 - 5 } & & Mean & \pm SD & \multicolumn{2}{|c|}{ Mean } \\
\hline \multirow{3}{*}{1 RM squat $(\mathrm{kg})$} & SES & 112.43 & 4.04 & $137.71^{*} \#$ & 3.25 \\
\cline { 2 - 6 } & ESS & 112 & 5.66 & $126.57^{*}$ & 4.89 \\
\cline { 2 - 6 } & ASBS & 113.67 & 5.28 & $133^{*}$ & 6.10 \\
\hline \multirow{3}{*}{1 RM bench press (kg) } & SES & 67.57 & 2.70 & $78^{*} \#$ & 2.58 \\
\cline { 2 - 6 } & ESS & 68.43 & 3.10 & $73.71^{*}$ & 3.30 \\
\cline { 2 - 6 } & ASBS & 69.67 & 2.07 & $76.17^{*}$ & 2.23 \\
\hline \multirow{3}{*}{ LME squat (reps) } & SES & 11.43 & 1.13 & $13.57^{*} \#$ & 0.36 \\
\cline { 2 - 6 } & ESS & 10.57 & 0.98 & $11.71^{*}$ & 0.95 \\
\cline { 2 - 6 } & ASBS & 11.67 & 1.03 & 1.27 \\
\hline \multirow{3}{*}{ LME bench press (reps) } & SES & 10.86 & 1.22 & $12.71^{*}$ & 0.76 \\
\cline { 2 - 6 } & ESS & 11 & 0.82 & $12.29^{*}$ & 0.49 \\
\cline { 2 - 6 } & ASBS & 11.17 & 0.75 & $12.67^{*}$ & 0.81 \\
\hline \multirow{2}{*}{4 km running time trail (sec) } & SES & 954.14 & 14.87 & $902^{*}$ & 13.77 \\
\cline { 2 - 6 } & ESS & 957.14 & 21.67 & $868.14^{*} \&$ & 30.48 \\
\cline { 2 - 6 } & ASBS & 959 & 19.44 & $891.50^{*}$ & 23.07 \\
\hline
\end{tabular}

Values were expressed by mean $\pm \mathrm{SD}(\mathrm{n} ; \mathrm{SES}=7, \mathrm{ESS}=7$, $\mathrm{ASBS}=6), 1 \mathrm{RM}=$ one repetition maximum; $\mathrm{LME}=$ Local muscular endurance; $\mathrm{PRE}=$ pre-training evaluation; $\mathrm{POST}=$ post-training evaluation; *statistically significant difference in comparison with PRE; \#statistically significant difference in comparison with ESS and ASBS; \& statistically significant difference in comparison with SES and ASBS $(\mathrm{p} \leq 0.05)$.

Figure 2

Rate of changes in $1 \mathrm{RM}(\mathrm{A}), \mathrm{LME}(\mathrm{B})$, and $4 \mathrm{~km}$ running time trail (C) for all groups

A

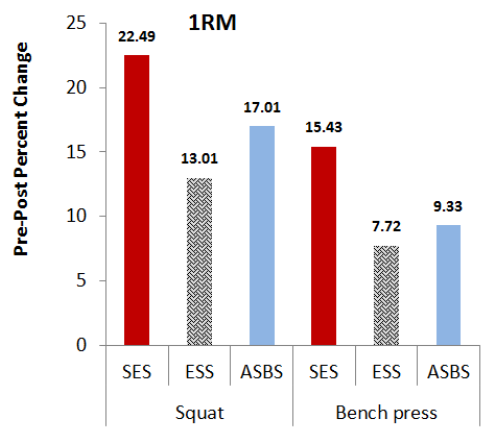

B

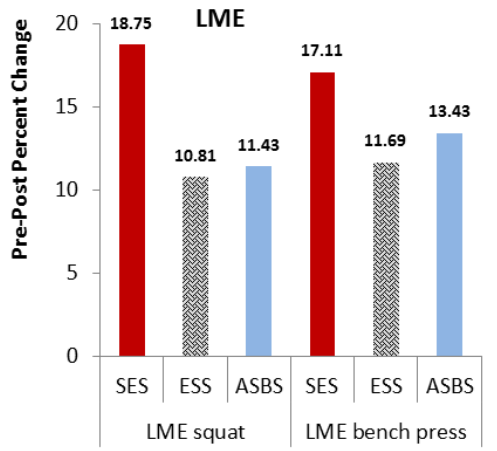

C

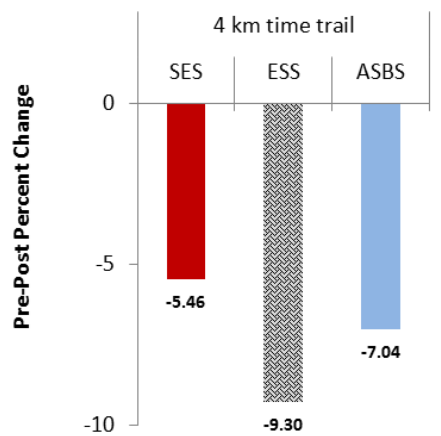

The LME; The study has shown statistically significant differences in LME of squat at the POST evaluation $(\mathrm{p} \leq$ 0.05 ) favor SES group of $18.72 \%$ in comparison with the ESS group of $10.78 \%$, while there were no statistically significant differences $(p>0.05)$ in comparison with the ASBS group of $11.40 \%$, moreover no statistically significant differences $(p>0.05)$ were found between ESS and ASBS groups, however the ASBS group shown a higher percentage increase in LME of squat exercise than ESS group (Table 4) (Fig. 2B). Whereas the study has shown no statistically significant differences in LME of bench press $(\mathrm{p}>0.05)$ between SES, ESS, and ASBS of $(17.03 \%, 11.72 \%$, and $13.42 \%$; respectively), however the SES group shown a highest percentage increase in LME of bench press, then ASBS group and finally ESS group (Table 4) (Fig. 2B) 


\section{Aerobic fitness}

$4 \mathbf{~ k m}$ running time trail: The study has shown statistically significant differences favor ESS group $(\mathrm{p} \leq 0.05)$ at the POST evaluation in $4 \mathrm{~km}$ running time trail of $-9.30 \%$, in comparison with the SES group of $-5.46 \%$, whereas there is no statistically significant differences $(\mathrm{p}>0.05)$ were found in comparison with the ASBS group of $-7.04 \%$. Also the study has shown no statistically significant differences $(p>0.05)$ between ASBS and SES groups in LME, however the ASBS group shown a higher percentage increase in aerobic endurance than SES group for those exercises (Table 4) (Fig. 2C).

\section{Discussion}

The current study is the first to investigate the sequence effect of concurrent strength and endurance training among volleyball players in three groups, one of them has alternate sequence of strength and endurance every session. The results demonstrate significant effects of CT with different sequence in a maximal strength and local muscular endurance for upper and lower body in addition to an aerobic fitness.

\section{Muscular fitness}

The main results confirmed that maximal strength and local muscular endurance were improved in squat and bench press for three experimental groups. Thus the 1st hypothesis was accepted, this result is consistent with several studies (M Chtara et al., 2005; Moktar Chtara et al., 2008; Enright, 2015; McCarthy, 2002; McGawley \& Andersson, 2013; Silva et al., 2012; Wong, 2010). Another finding of current study was a statistically significant differences favor SES group in 1RM of squat and bench press and in LME of squat for SES group than ESS group, so no interference was found in SES group and ASBS group, whereas a little interference was found in ESS group. Thus the 2nd hypothesis was accepted, this result is consistent with several studies (Alves, 2015; Enright, 2015; Glowacki et al., 2004; McCarthy, 2002). Strength training first sequence would favor gains in muscle strength, because of the absence of pre-exercise fatigue resulting from aerobic exercise. This sequence may be of particular interest to athletes whose sports demand strength and power, by placing strength sessions before the endurance sessions or, when not feasible, separating both types of training by at least 6 hours to allow for sufficient recovery (Kang \& Ratamess, 2014). A potential drawback of the endurance-first sequence is that endurance training if performed vigorously can compromise the performance of subsequent resistance exercise, thereby attenuating strength development during CT (Gravelle \& Blessing, 2000; Kraemer et al., 1995). It is believed that residual fatigue from the endurance component of CT can reduce the tension developed during the strength element of CT. Contrary to our results; several studies have interference in strength gain in their CT group (García-Pallarés \& Izquierdo, 2011; Hickson, 1980; Kraemer et al., 1995). The frequency of concurrent strength and endurance training has been shown to influence the adaptive response. When the frequency of concurrent strength and endurance training is high (4-6 days per week), a reduced improvement in muscular strength has been observed (Hickson, 1980; Kraemer et al., 1995). However, when the training frequency of concurrent strength and endurance training is low (2-3 days per week) as current study, maximal strength has been shown to increase at a rate similar to that of strength training alone. Another interesting finding in current study, the LME gain of bench press were almost similar in the three groups, it can be ascribed to the different muscle groups were used during endurance exercise, therefore did not affect the LME gain of upper limp, (Sporer \& Wenger, 2003) found that the bench press repetitions after the aerobic session was not reduced.

\section{Aerobic fitness}

The main results confirmed that aerobic fitness was improved in $4 \mathrm{~km}$ running time trail for three experimental groups. this result is consistent with study of (M Chtara et al., 2005), Another finding of current study was a statistically significant differences favor ESS group in 4 $\mathrm{km}$ running time trail than SES group, so no interference was found in ESS group and ASBS group, whereas a little interference was found in SES group. Thus the 3rd hypothesis was accepted, this result is consistent with several studies (M Chtara et al., 2005; Gravelle \& Blessing, 2000). Study of (M Chtara et al., 2005) reported that placing aerobic training before resistance training could improve running performance and $\mathrm{VO} 2 \mathrm{max}$ to a greater extent than the reverse order. Study of Drummond et al. 2005 (Drummond, 2005) found that the aerobic-first sequence was more effective in augmenting excess postexercise oxygen consumption. In this study, the aerobic session involved continuous running at a pace that corresponded to $70 \%$ VO2max for 25 minutes. Study of Davis et al. 2008 (Davis, 2008) used a "cardio acceleration" protocol in which subjects were asked to run for up to a minute at $60 \%$ to $84 \%$ of heart rate reserve immediately before each set of resistance exercise. They found that this cardio-first sequence was able to eliminate the delayed-onset muscle soreness by the fourth week of training.

One of the most interesting finding in the current study was the improvement of muscular and aerobic fitness in 
ASBS group was similar with SES and ESS groups, so the alternate sequence between sessions leaded to no interference effect on muscular and aerobic fitness, and make CT more effectively.

\section{Conclusion}

The Sequence of concurrent strength and endurance training influence the adaptive responses to them. Improvement in muscular fitness is significantly greater when, in the same session, the strength training precede the endurance training rather than the other way around. Improvement in endurance performance is significantly greater when, in the same session, the endurance training precede the strength training rather than the other way around. Alternately Performance for strength and endurance training every session seems more effective in enhancing muscular and aerobic fitness alike.

\section{References}

1. Alves, A. R. M., Carlos Neiva, Henrique P Izquierdo, Mikel Marques, Mário C. (2015). Concurrent training in prepubescent children: the effects of eight weeks of strength and aerobic training on explosive strength and VO2max. Journal of strength and conditioning research/National Strength \& Conditioning Association.

2. Atherton, P. J., Babraj, J., Smith, K., Singh, J., Rennie, M. J., \& Wackerhage, H. (2005). Selective activation of AMPK-PGC-1 $\alpha$ or PKB-TSC2-mTOR signaling can explain specific adaptive responses to endurance or resistance training-like electrical muscle stimulation. The FASEB journal, 19(7), 786-788.

3. Baar, K. (2006). Training for endurance and strength: lessons from cell signaling. Medicine and science in sports and exercise, 38(11), 1939.

4. Baker, D., Wilson, G., \& Carlyon, R. (1994). Periodization: The Effect on Strength of Manipulating Volume and Intensity. The Journal of Strength \& Conditioning Research, 8(4), 235-242.

5. Bell, G. S., D Martin, TP Burnham, R Quinney, HA. (2000). Effect of concurrent strength and endurance training on skeletal muscle properties and hormone concentrations in humans. European journal of applied physiology, 81(5), 418-427.

6. Chtara, M., Chamari, K., Chaouachi, M., Chaouachi, A., Koubaa, D., Feki, Y., . . . Amri, M. (2005). Effects of intra-session concurrent endurance and strength training sequence on aerobic performance and capacity. British journal of sports medicine, 39(8), 555-560.

7. Chtara, M., Chaouachi, A., Levin, G. T., Chaouachi, M., Chamari, K., Amri, M., \& Laursen, P. B. (2008).
Effect of concurrent endurance and circuit resistance training sequence on muscular strength and power development. The Journal of Strength \& Conditioning Research, 22(4), 1037-1045.

8. Davis, W. J., Wood, Daniel T, Andrews, Ryan G, Elkind, Les M, Davis, W Bart. (2008). Elimination of delayed-onset muscle soreness by pre-resistance cardioacceleration before each set. The Journal of Strength \& Conditioning Research, 22(1), 212-225.

9. Docherty, D., \& Sporer, B. (2000). A proposed model for examining the interference phenomenon between concurrent aerobic and strength training. Sports Medicine, 30(6), 385-394.

10. Drummond, M. J., Vehrs, Pat R, Schaalje, G Bruce, Parcell, Allen C. (2005). Aerobic and resistance exercise sequence affects excess postexercise oxygen consumption. The Journal of Strength \& Conditioning Research, 19(2), 332-337.

11. Enright, K. M., James Iga, John Drust, Barry. (2015). The effect of concurrent training organisation in youth elite soccer players. European journal of applied physiology, 115(11), 2367-2381.

12. Fyfe, J. J., Bishop, D. J., \& Stepto, N. K. (2014). Interference between concurrent resistance and endurance exercise: molecular bases and the role of individual training variables. Sports Medicine, 44(6), 743-762.

13. García-Pallarés, J., \& Izquierdo, M. (2011). Strategies to optimize concurrent training of strength and aerobic fitness for rowing and canoeing. Sports Medicine, 41(4), 329-343.

14. Glowacki, S., Martin, S., Maurer, A., Baek, W., Green, J. S., \& Crouse, S. F. (2004). Effects of resistance, endurance, and concurrent exercise on training outcomes in men. Medicine and science in sports and exercise, 36, 2119-2127.

15. Gravelle, B., \& Blessing, D. (2000). Physiological Adaptation in Women Concurrently Training for Strength and Endurance. The Journal of Strength \& Conditioning Research, 14(1), 5-13.

16. Hickson, R. C. (1980). Interference of strength development by simultaneously training for strength and endurance. European journal of applied physiology and occupational physiology, 45(2-3), 255-263.

17. Izquierdo, M., \& Exposito, R. G.-P., J Medina, L Villareal, E. (2010). Concurrent endurance and strength training not to failure optimizes performance gains. Sci Sports Exerc, 42, 1191-1199.

18. Janssen, P. (2001). Training at anaerobic threshold. Lactate threshold training, 2nd ed. United States: Human Kinetics, 4, 25-41. 
19. Kang, J., \& Ratamess, N. (2014). Which Comes First? Resistance Before Aerobic Exercise or Vice Versa? ACSM's Health \& Fitness Journal, 18(1), 9-14.

20. Karvonen, M. J., E. Kentala, and O. Mustala. (1957). The effects of training on heart rate. Inst. Occupational Health, 307-315.

21. Kraemer, W. J., Patton, J. F., Gordon, S. E., Harman, E. A., Deschenes, M. R., Reynolds, K., . . D Dziados, J. E. (1995). Compatibility of high-intensity strength and endurance training on hormonal and skeletal muscle adaptations. Journal of applied physiology, 78(3), 976989.

22. Marques, M. C., Van den Tillaar, Roland Gabbett, Tim J Reis, Victor M, González-Badillo, Juan J. (2009). Physical fitness qualities of professional volleyball players: determination of positional differences. The Journal of Strength \& Conditioning Research, 23(4), 1106-1111.

23. McCarthy, J. P. P., Myron A Agre, James C. (2002). Neuromuscular adaptations to concurrent strength and endurance training. Medicine and science in sports and exercise, 34(3), 511-519.

24. McGawley, K., \& Andersson, P.-I. (2013). The order of concurrent training does not affect soccer-related performance adaptations. Int J Sports Med, 34(11), 983990.

25. Mikkola, J., Rusko, H, Nummela, A, Pollari, T, Hakkinen, K. (2007). Concurrent endurance and explosive type strength training improves neuromuscular and anaerobic characteristics in young distance runners. International journal of sports medicine, 28(7), 602-611.
26. Quebedeaux, L. P. (2007). The effect of two concurrent training programs with different inter-session recovery on musculoskeletal strength [electronic resource]. UMI thesis.

27. Santos, A. P. M., Daniel A Costa, Aldo M, \& Izquierdo, M. M., Mário C. (2012). The effects of concurrent resistance and endurance training follow a detraining period in elementary school students. The Journal of Strength \& Conditioning Research, 26(6), 1708-1716.

28. Silva, R., Cadore, E., Kothe, G., Guedes, M., Alberton, C., Pinto, S., . . . Kruel, L. (2012). Concurrent Training with Dịerent Aerobic Exercises. Int J Sports Med, 33, 627634.

29. Sporer, B. C., \& Wenger, H. A. (2003). Effects of aerobic exercise on strength performance following various periods of recovery. The Journal of Strength \& Conditioning Research, 17(4), 638-644.

30. Wilson, J. M., Marin, P. J., Rhea, M. R., Wilson, S. M., Loenneke, J. P., \& Anderson, J. C. (2012). Concurrent training: a meta-analysis examining interference of aerobic and resistance exercises. The Journal of Strength \& Conditioning Research, 26(8), 2293-2307.

31. Wong, P.-1., Chaouachi, Anis, Chamari, Karim, Dellal, Alexandre, Wisloff, Ulrik. (2010). Effect of preseason concurrent muscular strength and high-intensity interval training in professional soccer players. The Journal of Strength \& Conditioning Research, 24(3), 653-660. 